\title{
Search for giant extrasolar planets around white dwarfs: direct imaging with NICMOS/HST and NACO/VLT
}

\author{
Hans Zinnecker ${ }^{1}$, Serge Correia ${ }^{1}$, Wolfgang Brandner ${ }^{2}$, \\ Susanne Friedrich ${ }^{3}$, and Mark McCaughrean ${ }^{4}$ \\ ${ }^{1}$ Astrophysikalisches Institut Potsdam, An der Sternwarte 16, D-14482 Potsdam, Germany \\ email: hzinnecker@aip.de \\ email: scorreia@aip.de \\ ${ }^{2}$ Max-Planck-Institut für Astronomie, Auf dem Königsstuhl, D-69117 Heidelberg, Germany \\ email: brandner@mpia.de \\ ${ }^{3}$ Max-Planck-Institut für Extraterrestrische Physik, Giessenbachstr., \\ D-85748 Garching, Germany \\ email: sfriedr@mpe.mpg.de \\ ${ }^{4}$ Astrophysics Group, School of Physics, University of Exeter, Stocker Road, \\ Exeter EX4 4QL, UK \\ email:mjm@astro.ex.ac.uk
}

\begin{abstract}
We report preliminary results from our search for massive giant planets (6-12 Jupiter masses) around the known seven single white dwarfs in the Hyades cluster at sub-arcsec separations. At an age of $625 \mathrm{Myr}$, the white dwarfs had progenitor masses of about 3 solar masses, and massive gaseous giant planets should have formed in the massive circumstellar disks around these ex-Herbig A0 stars, probably at orbital separations similar or slightly larger than that of Jupiter. Such planets would have survived the post-Main-Sequence mass loss of the parent star and would have migrated outward adiabatically to orbital separations of about $25 \mathrm{AU}$. At the distance of the Hyades ( $45 \mathrm{pc}$ ) this corresponds to angular separations of about 0.5 arcsec which can be resolved with NICMOS/HST; the expected contrast in the $\mathrm{J}$ and $\mathrm{H}$ bands amount to $7.5 \pm 1.5 \mathrm{mag}$.

Evaluation of our NICMOS data set did not reveal any evidence for planetary mass companions with masses down to about 10 Jupiter masses nor brown dwarfs around any of the seven white dwarfs for separations larger than 0.5 arcsec. However, we detected a low-mass, probably stellar, companion to a field white dwarf (WD1847-223J, distance $\sim 50 \mathrm{pc}$, age $\sim 1 \mathrm{Gyr}$; separation $\sim 0.5 \operatorname{arcsec}, \Delta \mathrm{H} \sim 2.5 \mathrm{mag}$ ), using the NACO adaptive optics system at the VLT.
\end{abstract}

Keywords. extrasolar planets, white dwarfs, imaging, HST, VLT.

\section{Planets around White Dwarfs}

Since the discovery of the first extrasolar planet by Mayor \& Queloz (1995) the number of known extrasolar planets has grown to more than 150. Most discoveries are based on measurements of radial velocities of stellar lines. Due to the huge contrast in brightness between the main sequence star and its planet(s) no planet has so far been seen directly with the possible exception of a planetary-mass companion to a young brown dwarf (Chauvin et al. 2005). The contrast problem can be reduced if one searches for planets around white dwarfs, which are about a factor of ten smaller than giant planets $\left(F \sim R^{2}\right)$ and typically $10^{3}$ to $10^{4}$ times less luminous than main sequence stars of spectral type G or K. 
For our search we have chosen the only young cluster in the near vicinity with a large enough sample of white dwarfs: the Hyades. Compared to isolated white dwarfs the Hyades cluster white dwarfs have the advantage that their age is known from the turnoff age of the cluster (625 Myr, Perryman et al. 1998). This also implies that any planet will be of the same age. A Hyades main sequence star with $3 \mathrm{M}_{\odot}$ (the turn-off mass) will eventually become a $0.66 \mathrm{M}_{\odot}$ white dwarf (Weidemann 2000). All single stars with masses above the turn-off mass and below about $8 \mathrm{M}_{\odot}$ have already left the main sequence and will develop into or have already become white dwarfs. While planets closer than $3-5 \mathrm{AU}$ will probably not survive this post-main-sequence evolution (and will migrate inwards and merge) planets farther away will survive (Duncan \& Lissauer 1998; Burleigh et al. 2002; Rasio et al. 1996), and their semimajor axis will increase by a factor $M_{\text {initial }} / M_{\text {final }}$ as the central star loses mass. Thus any giant planet in a Jupiter-like orbit ( $\geqslant 5$ AU) will migrate outwards to a new equilibrium radius of at least:

$$
\mathrm{R}=5 \mathrm{AU} \times\left(3 \mathrm{M}_{\odot} / 0.66 \mathrm{M}_{\odot}\right) \approx 22.5 \mathrm{AU}
$$

At a distance of the Hyades (45 pc) 22.5 AU correspond to 0.5 arcsec. Furthermore, a giant planet with mass somewhat below 13 Jupiter masses (which is one of the definition for "planet") orbiting a white dwarf in the Hyades has a near-infrared magnitude difference of only about $\mathbf{7 . 5}$ mag. Resolving such a favorable contrast at such a favorable angular separation is feasible with NICMOS in the F110W and F160W filters with spatial resolutions of 0.1 arcsec and 0.15 arcsec, respectively, as our simulations have shown. The same is true for near-infrared direct imaging with the NACO adaptive optics system at the VLT.

\section{Observations and Data Reduction}

The seven single white dwarfs (HZ4, LB227, VR7, VR16, HZ7, HZ14, LP475-242) have been observed with HST/NICMOS at two roll angles separated by 20 degrees through the F110W and F160W filters (program GO 9737). Two and four dithered frames, respectively, of $320 \mathrm{~s}$ integration time each were recorded for each roll angle and each target in these filters, which corresponds to a total of one and two orbits of HST time per target, respectively. The single dithered frames were reduced using the HST pipeline, then oversampled by a factor two using cubic spline before registration and a FFT-shift based combination for each roll angle. Subsequent bad-pixel masking was further applied in order to compensate for the non-optimal bad-pixel mask available through the pipeline. For that purpose, remaining not corrected bad-pixels were identified using a criterion based on the deviation from the median of all frames available for each target. In each filter, the resulting frames corresponding to different roll angles were subtracted from each other. This step was done after the use of a $2 \mathrm{D}$ cross-correlation for the registration of these frames, which provided a sub-pixel accuracy, and a FFT-shift based combination. The result obtained for HZ7 is presented in Fig. 1 as an example.

The NACO/VLT observations were obtained on 2004, May 3, in service mode in relatively good seeing $(0.5-0.7$ arcsec). The resulting ESO pipeline $\mathrm{H}$-band image is presented in Fig. 4.

\section{Method Validation and Contrast Ratio}

We validated the method used in the data reduction through simulations. For this purpose a fake companion of given brightness was introduced in each frame as a scaled version of the target, adding the appropriate noise, and checking that we could detect the signature of the companion after all reduction steps described above. Fig. 2 shows the 

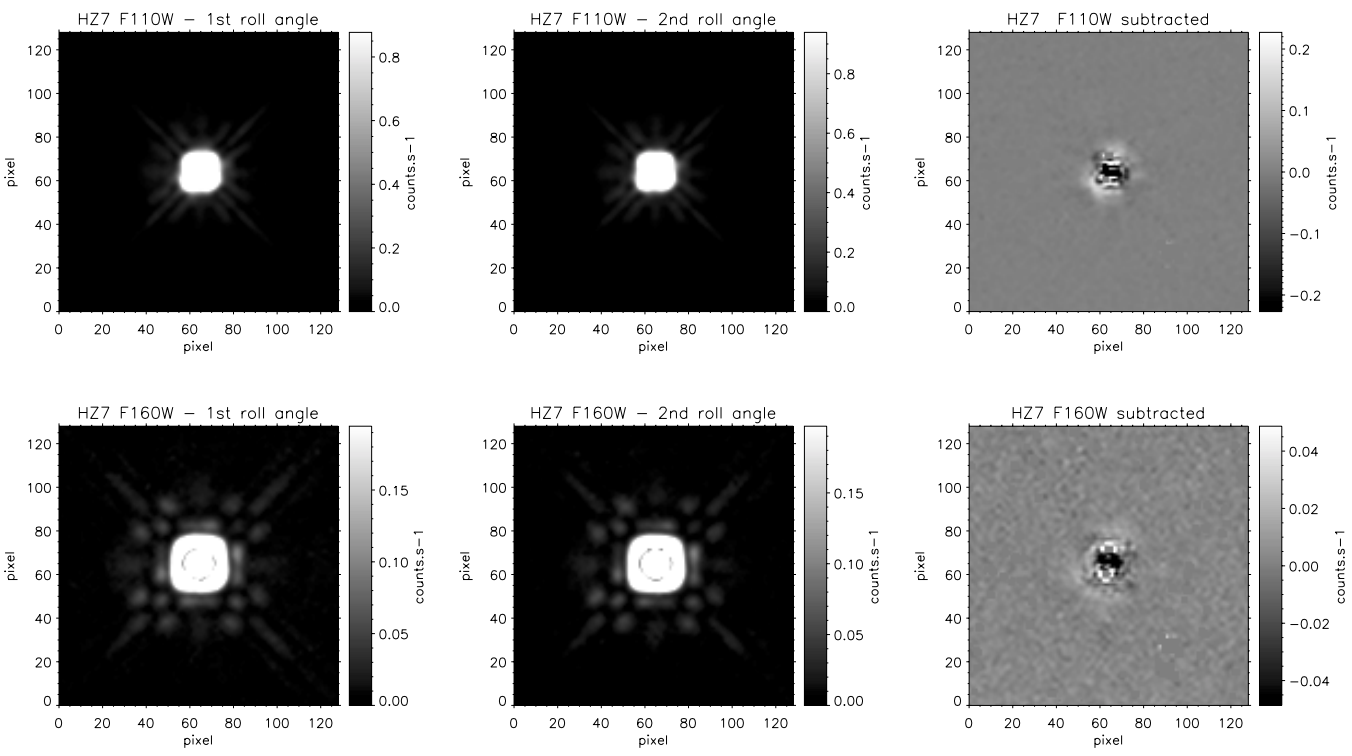

Figure 1. The observations of HZ7 at two different roll angles and the result of their subtractions in the F110W filter (upper panel) and the F160W filter (lower panel). The field of view is about $2.75 \operatorname{arcsec} \times 2.75 \operatorname{arcsec}(0.0215 \operatorname{arcsec}$ pixel size $)$. Maximum cuts are at $2 \%$ of the peak intensity for the observations, and $\pm 0.5 \%$ for the subtracted frames.
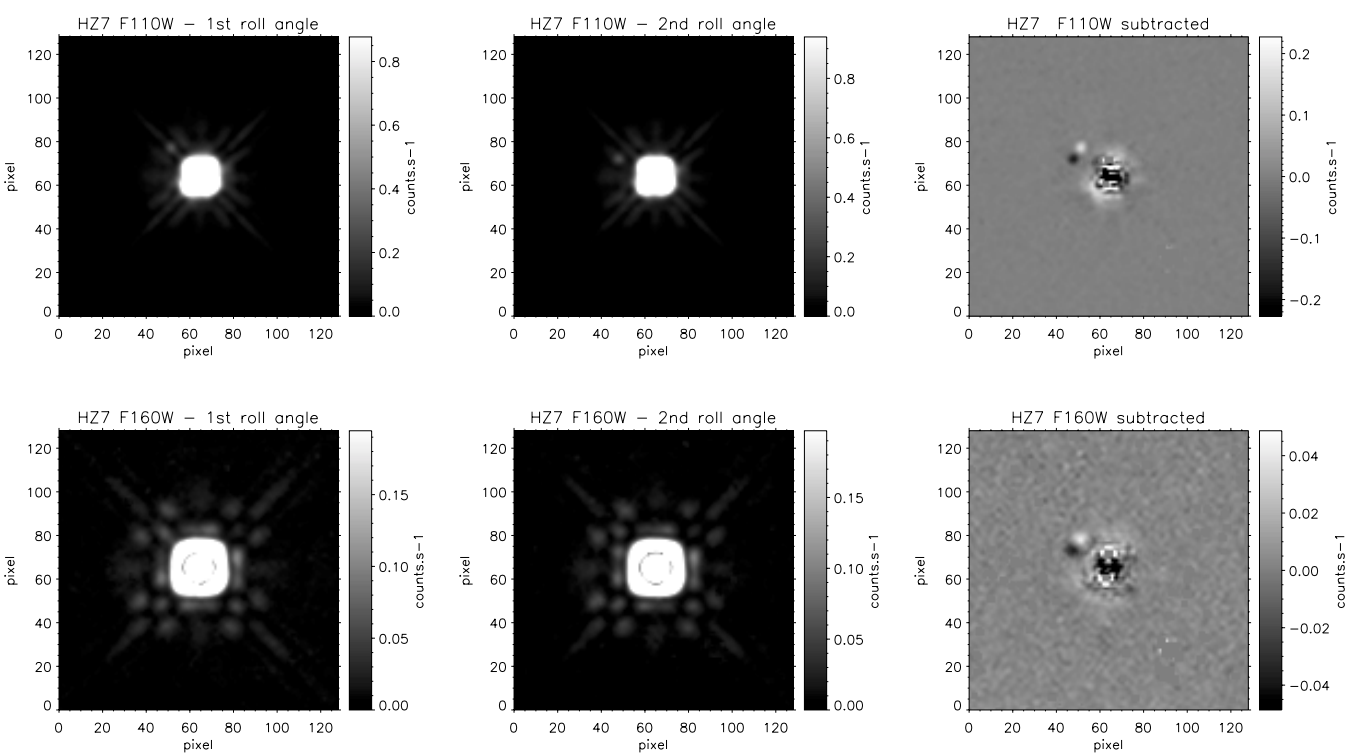

Figure 2. Same as Fig. 1 with an artificial planetary mass companion added (6 mag brightness difference, 0.4 arcsec separation).

results of such a simulation for both filters and the same target as in Fig. 1, with a planet at 0.4 arcsec separation and 6 magnitudes difference in brightness. One can easily notice the pair of negative and positive images of the companion at the given position from the star in the subtracted frame. Such a simulation allows to estimate the limiting difference 
in brightness as a function of separation and given SNR of the detection. Fig. 3 shows the result for the same target as in Fig. 1 in each filter and a detection threshold of $5 \sigma$. The uncertainties are estimated from the scatter obtained using four different position angles of the companion. According to the COND models of Baraffe et al. (2003), the limiting mass of the planetary mass companion that we are able to detect is typically of about 10 Jupiter masses at 0.5 arcsec separation (Friedrich et al. 2005).
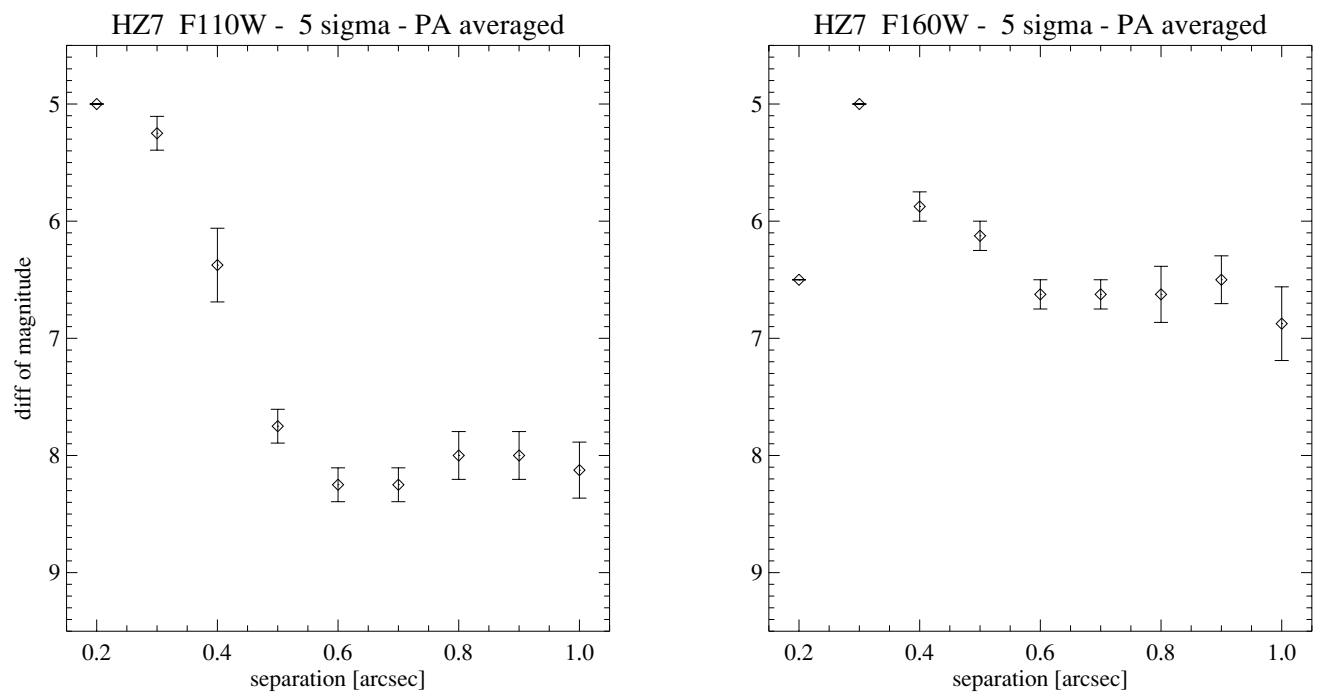

Figure 3. Estimated sensitivity limits as a function of separation for HZ7. Uncertainties are estimated from the scatter obtained using four different position angles of the companion.

\section{Conclusion and Discussion}

We did not find any evidence for neither planetary mass companions with masses down to about 10 Jupiter masses nor brown dwarfs around any of the seven white dwarfs in the Hyades for separations larger than 0.5 arcsec. Possible reasons for the non-detection include

- small number statistics (giant $\sim 10 \mathrm{M}_{J u p}$ planets are rare, $<10 \%$ )

- limited sensitivity (can’t detect $\sim 1 \mathrm{M}_{J u p}$ planets yet, too faint)

- hot planetary nebula (X-rays) may have evaporated the giant planet

- planet-planet perturbations during mass loss unbinds giant planets

- original giant planet has spiralled in (gas drag) and was swallowed

At present, we cannot decide whether our null result is due to the non-existence of giant planets in orbit around the progenitor stars or due to them not surviving postMain Sequence evolution. The latter seems more likely (Villaver \& Livio 2005, private communication; Debes \& Sigurdsson 2002, Duncan \& Lissauer 1998), especially if the giant planets have small orbital radii. In this context, we note that a giant planet of $\mathrm{M} \sin i=6.5 \mathrm{M}_{J u p}$ with semi-major axis of some $2 \mathrm{AU}$ and eccentricity $\mathrm{e}=0.4$ has been found by the radial velocity technique around a G5 red giant star (HD 11977) of about $2 \mathrm{M}_{\odot}$, i.e. an A-star Main Sequence progenitor (Setiawan et al. 2005); thus giant planets appear to exist around early-type progenitors like our Hyades stars (good news), but it is clear that this giant planet is in danger to spiral into the red giant envelope and get lost (bad news). Indeed, we made a simple estimate, based on the Bondi-Hoyle formalism, 
how long it takes for a $5 \mathrm{M}_{J u p}$ planet on a circular orbit of radius $1 \mathrm{AU}$ to spiral into a red giant of $3 \mathrm{M}_{\odot}$, given that the envelope mass density at $1 \mathrm{AU}$ is $10^{-7} \mathrm{gcm}^{-3}$ (Herwig, private communication). The answer is about $100 \mathrm{yr}$ (this is actually the timescale on which the giant planet is going to double its own mass by envelope gas accretion, and the corresponding accretional drag will make the orbit decay on the same timescale).

\section{Outlook}

Despite the NICMOS/HST non-detection, the direct imaging search for giant planets around white dwarfs is a promising avenue and should be further pursued in the future, e.g. with JWST. Meanwhile, the next step is to search for mid-infrared excess on white dwarfs caused by thermal emission of the planets, using the Spitzer Space Telescope camera (IRAC). Although its angular resolution is only 1.5 arcsec, implying that any detection would likely be spatially unresolved, its sensitivity is such that we could see giant planets at 4.5 microns with masses of 2 to $3 \mathrm{M}_{\text {Jup }}$ around nearby young hot white dwarfs, such as WD $1134+300$ at a distance of $\sim 15 \mathrm{pc}$ and an age of $\sim 0.2 \mathrm{Gyr}(25-50 \%$ excess emission). Spitzer observing programmes are underway, including one by our team (no results yet).

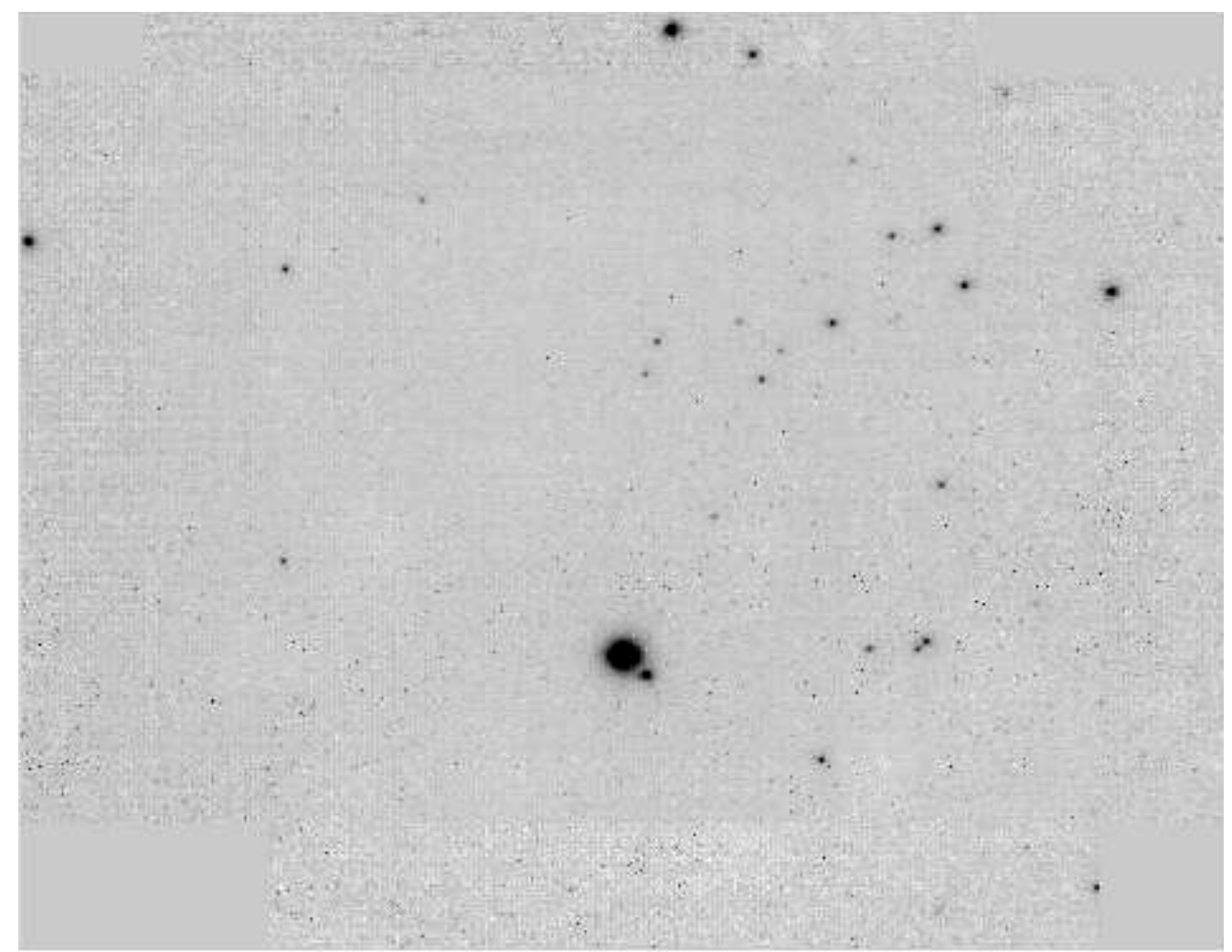

Figure 4. NACO/VLT H-band image of the field white dwarf WD1847-223J showing a $\sim 10$ times fainter companion towards the south-west (separation $\sim 0.5 \operatorname{arcsec}$ ). 


\section{Acknowledgements}

We thank Matt Burleigh for sharing with us his calculations of the detectability of giant planets around white dwarfs through mid-infrared Spitzer observations. We also thank Eva Villaver for pointing out the possibility of X-ray evaporation of giant planets during the hot planetary nebula phase. Furthermore, thanks are due to Falk Herwig for making his red giant structural models available. Last but not least, congratulations to the organizers for a wonderful last IAU Colloquium!

\section{References}

Baraffe, I., Chabrier, G., Barman, T.S., Allard, F., \& Hauschildt, P.H. 2003, A\& $A$ 402, 701

Burleigh, M.R., Clarke, F.J., \& Hodgkin, S.T. 2002, MNRAS 331, L41

Chauvin, G., Lagrange, A.-M., Dumas, C., Zuckerman, B., Mouillet, D., Song, I., Beuzit, J.-L., \& Lowrance, P. 2005, A\&A (Letters) 438, L25

Debes, J.H. \& Sigurdsson, S. 2002, ApJ 572, 556

Duncan, M.J. \& Lissauer, J.J. 1998, Icarus 134, 303

Friedrich, S., Zinnecker, H., Brandner, W., Correia, S., \& McCaughrean, M. 2005, astro$\mathrm{ph} / 0501148$

Mayor, M. \& Queloz, D. 1995, Nature 378, 355

Neuhäuser, R., Guenther, E.W., Wuchterl, G., Mugrauer, M., Bedalov, A., \& Hauschildt, P.H. 2005, A\&\&A (Letters) 435, L13

Perryman, M.A.C., Brown, A.G.A., Lebreton, Y. Gomez, A., Turon, C., de Strobel, G.C., Mermilliod, J.C., Robichon, N., Kovalevsky, J., \& Crifo, F. 1998, A\&A 331, 81

Rasio, F.A., Tout, C.A., Lubow, S.H., Livio, M. 1996, ApJ 470, 1187

Setiawan, J., Rodmann, J., da Silva, L. et al. 2005 A $\& A$ 437, L31

Weidemann, V. 2000, A\& A 363, 64 